\title{
Geometrical Channel Characterization for High Speed Railway Environments Using Propagation Graphs Methods
}

\author{
Wenpu Cheng*, Cheng Tao*, Liu Liu**, Rongchen Sun*, Tao Zhou* \\ ${ }^{*}$ Institute of Broadband Wireless Mobile Communications, Beijing Jiaotong University, Beijing 100044, P. R. China \\ ${ }^{* *}$ National Mobile Communications Research Laboratory, Southeast University, Nanjing 210096, P.R.China \\ Email: $\{11120070$, chtao, liuliu, 11111036, 11111037\}@bjtu.edu.cn
}

\begin{abstract}
In this paper, a new geometry-based channel characterization technique for the high-speed railway scenarios is employed by using the graphic methods. The multi-dimensional channel parameters, such as time delay and Doppler property, can be extracted analytically for the specific environment. By using the graph modeling approach, the channel impulse responses (CIRs) can be calculated by searching for propagation paths, which provide a solid theoretical foundation for high-quality research of ultra-high-speed mobile communications. Then Von Mises distribution is used to model the non-uniform Angle-ofArrival (AoA) at Rx, and the simulation results can be applied to characterize the feasibility of the theoretical channel modeling method. Finally, the established model is compared with the actual measurement data in cutting scenario. The statistical results from the proposed model including the time-varying power delay profile and rapid Doppler transition show a good match with the experimental data, which shows that the study of the proposed modeling method is significant.
\end{abstract}

Index Terms-High-Speed Railway; Von Mises; Propagation Graph

\section{INTRODUCTION}

Regardless of the transmission technique employed, the knowledge of the wireless channel is vital to the optimal design and performance of any communication system. Much of the previous literature on the channel measurement and characterization is for terrestrial cellular communication systems. Since the 1940s, researchers conducted intensive studies for wireless channel of various propagation environments, then the results achieved are actually contributed to the development of wireless communication greatly. COST project-207 proposed by European Cooperation in Science and Technology is widely used in simulation assessment for GSM system [1], then broadband models SCM (Spatial Channel Model) based on geometrical-cluster and Winner II, [2] [3], including urban hot spots, Coordinated Multiple Points (CoMP), high-speed railway (HSR), etc. are successfully used in evaluation and experiment for multi-antenna LTE (Long Term Evolution) and LTE-A (Long Term Evolution-Advanced).

Broadband wireless communications in HSR scenarios are expected to offer reliable and high-capacity network between the high speed train and the way-side [4][5]. To cope with problems faced by the development of HSR wireless transmission systems, novel propagation channel models are inevitably required to make a detailed knowledge about their statistical properties.Actually, there are considerable difficulties to conduct the measurement experiment in HSR environments, such as strict limitation of frequency bands, extremely lower measurement efficiency and costly reservation for the test train. Therefore, most propagation characteristics under the typical HSR environments are still unknown and have thus to be derived from scratch. Starting from specific geometrical scattering models, we employ a novel channel characterization technique based on propagation graphs methods to prove the typical plain channel in HSR environments.

This propagation graph channel modeling method was firstly published in Vienna Symposium on Mathematical Modeling in [6]. It established the simulated reflectors, scattering objects, and other physical phenomena in the propagation environment so that radio waves experienced as the relation between vertices and edges. Yin [7] studied the correlation of angular fading in Non-Line-of-Sight (NLOS) scenarios, Bernard [8] exploited propagation characteristics of indoor layout for ray signature using channel modeling method based on propagation graph. Tian [9] proposed a channel modeling method based on random propagation graph to characterize the time-variant channel in typical high-speed railway wireless communication scenarios. So far, we have not got the research results of modeling method based on propagation graph in the moving channel environment.

The main contribution of this paper is to study the channel characteristics and modeling method based on propagationgraph theory for HSR scenarios. Particularly, fading characteristics in time, frequency and spatial domain will be analyzed. Furthermore, by using the graph modeling approach, the actual measurement data is adopted to parameterize the simulation results.

The remainder of the paper is organized as follows. In Section II, we describe the modeling approach of wireless channel based on propagation graph. In Section III, Von Mises distribution is employed to characterize the availability and feasibility of modeling method. Section IV provides the measurement data compared with the simulation results under high-speed railway scenarios to illustrate the application of the 
proposed model. The conclusion is drawn in Section V.

\section{Modeling Method Based on Propagation Graph}

As seen in Fig.1, the propagation environment can be described as a directed graph in which the vertices represent the transmitters, receivers and scatterers, and the edges stand for propagation paths between the vertices. Each propagation path can be characterized as an edge in this graph.

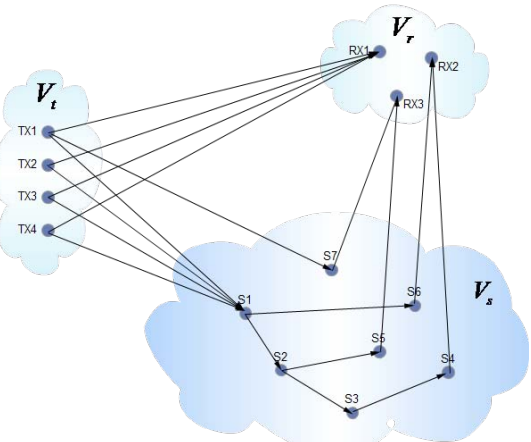

Fig. 1. A propagation graph with four transmit vertices, three receive vertices and seven scatterer vertices.

According to physical propagation mode, the transfer function of edge takes the form as

$$
A_{e}(f)=g_{e}(f) \cdot \exp \left(j\left(2 \pi \tau_{e} f+\phi_{e}\right)\right), e \in \varepsilon
$$

where $\tau_{e}$ and $\phi_{e}$ respectively denote the delay and random phase rotation of a given path tap, and $g_{e}$ is the gain of the propagation path. The propagation time $\tau_{e}$ of a signal propagating along edge $e=\left(v, v^{\prime}\right)$ can be calculated from the coordinates of $v$ and $v^{\prime}$ as $\tau_{e}=\frac{\left|\mathbf{r}_{v}-\mathbf{r}_{v^{\prime}}\right|}{c}$, where $c \approx 3 \cdot 10^{8} \mathrm{~m} / \mathrm{s}$ is the speed of light (in vacuum).

$g_{e}$ can be expressed as (reasonably modified for some parameters in literature [9], [7])

$$
g_{e}^{2}(f)= \begin{cases}\frac{1}{\left(4 \pi f \tau_{e}\right)^{2}} ; & e \in \varepsilon_{d} \mathrm{Tx} \rightarrow \mathrm{Rx} \\ \left(\frac{1}{4 \pi f \mu\left(\varepsilon_{t}\right)}\right)^{2} \cdot \frac{\tau_{e}-2}{S\left(\varepsilon_{t}\right)} ; & e \in \varepsilon_{t} \mathrm{Tx} \rightarrow \mathrm{S} \\ \left(\frac{1}{4 \pi f \mu\left(\varepsilon_{r}\right)}\right)^{2} \cdot \frac{\tau_{e}-2}{S\left(\varepsilon_{r}\right)} ; & e \in \varepsilon_{r} \mathrm{~S} \rightarrow \mathrm{Rx} \\ \frac{g^{2}}{\text { odi(e) }} ; & e \in \varepsilon_{s} \mathrm{~S} \rightarrow \mathrm{S}\end{cases}
$$

For any $\varepsilon^{\prime} \subseteq \varepsilon$

$$
\mu\left(\varepsilon^{\prime}\right)=\frac{1}{\left|\varepsilon^{\prime}\right|} \sum_{e \in \varepsilon^{\prime}} \tau_{e}, \quad S\left(\varepsilon^{\prime}\right)=\sum_{e \in \varepsilon^{\prime}} \tau_{e}^{-2}
$$

with $|\cdot|$ denoting cardinality, i.e. number of elements in the set. Therefore, $\mu\left(\varepsilon^{\prime}\right)$ denotes average propagation delay from transmitters vertices to scatterers; $\frac{\tau_{e}{ }^{-2}}{S(\varepsilon)}$ is the distance modified factor, describe the influence on $\left(\frac{1}{4 \pi f \mu(\varepsilon)}\right)^{2}$, i.e. the closer the distance, the greater the gain, also the greater the proportion of gain, and it is inversely proportional to the square of propagation time.

In addition, $\frac{g^{2}}{\text { odi(e) }}$ is power gain from scatterers to scatterers vertices, odi $(e)$ denotes the number of edges from a scatterer to other scatterers. This is a simplification of the energy conservation law, while considers that energy gain of internal scatterers has nothing to do with propagation, only associates with a number of reflected edges.

Thus, supposing we know the position of the scatters around the channel, we shall obtain the transfer function, after Inverse Fast Fourier Transform (IFFT), we have the CIRs.

The transfer function of channel has a closed-form expression as

$$
\mathbf{H}(f)=\mathbf{D}(f)+\mathbf{R}(f)[1-\mathbf{B}(f)]^{-1} \mathbf{T}(f)
$$

Where $\mathbf{D}(f)$ represents the Line-of-Sight (LOS) part of the transmission, $\mathbf{T}(f)$ denotes the matrix with each entry expressing the power attenuation and a random phase change from each transmitter (Tx) to each scatterer, $\mathbf{R}(f)$ denotes the channel coefficient matrix from the scatterers to the receivers $(\mathrm{Rx}), \mathbf{B}(f)$ refers to the matrix for inter-reflections of the scatterers. The closed form expression in (3) for the transfer matrix of propagation graph accounts for propagation via an arbitrary number of scatterer interactions. For some applications it is, however, pertinent to study only some part of the impulse response associated with a particular number of interactions. In the following we derive a few useful expressions for such partial transfer matrices.

We define the $\mathrm{K}: \mathrm{L}$ partial transfer matrix as

$$
\mathbf{H}_{\mathrm{K}: \mathrm{L}}(f)=\sum_{k=K}^{L} \mathbf{H}_{k}(f), 0 \leq K \leq L
$$

i.e., we include only contributions from propagation paths with at least $\mathrm{K}$, but no more than $\mathrm{L}$ bounces. On the other hand, if the bounce $\mathrm{L}$ is finite, then the partial response in (3) can be rewritten as

$$
\mathbf{H}_{\mathrm{K}: \mathrm{L}}(f)=\left\{\begin{array}{c}
\mathbf{D}(f)+\mathbf{R}(f)\left[\mathbf{I}-\mathbf{B}^{\mathrm{L}}(f)\right][\mathbf{I}-\mathbf{B}(f)]^{-1} \mathbf{T}(f), \\
K=0, L \geq 0 \\
\mathbf{R}(f)\left[\mathbf{B}^{K-1}(f)-\mathbf{B}^{\mathrm{L}}(f)\right][\mathbf{I}-\mathbf{B}(f)]^{-1} \mathbf{T}(f), \\
0<K \leq L
\end{array}\right.
$$

provided that the spectral radius of $\mathbf{B}(f)$ is less than unity.

Readers can refer to [8] and [10] for more information about partial transfer matrices of propagation graph modeling.

\section{The Characterization of Modeling Method}

In this section, we will show the validation of this theoretical channel modeling method. The statistical results will be compared with the measurement data. We have described the results from resent wideband channel measurement campaigns which are useful for the emulation and evaluation of wireless communication in typical HSR propagation scenarios. Details of the experimental equipment and methodology, the method to process the data, the method to extract model parameters from the measured data are presented in [12]. The complex envelope of the signal observed at Rx side can be decomposed as numerous accumulation of the exponential function,

$$
r^{s c a t}(t)=\lim _{\mathrm{N} \rightarrow \infty} \sum_{n=1}^{N} c_{n} \exp \left[j\left(2 \pi f_{n} t+\theta_{\mathrm{n}}\right)\right]
$$


Autocorrelation of $r^{s c a t}(t)$ can be written as [11]

$$
\begin{gathered}
\phi_{r r}^{\text {scat }}(\tau)=\lim _{\mathrm{N} \rightarrow \infty} \sum_{n=1}^{N} c_{n}^{2} E\left\{\exp \left(j 2 \pi f_{n} \tau\right)\right\} \\
E\left\{\exp \left(j 2 \pi f_{n} \tau\right)\right\}=\int_{-\infty}^{\infty} p_{f}(f) \exp (j 2 \pi f \tau) d f
\end{gathered}
$$

where $p_{f}(f)$ represents the probability density function (PDF) of the AoA.

Here, Von Mises distribution is used to characterize the feasibility of wireless channel modeling method based on graph theory. A realistic non-isotropic scattering model is introduced, and the correlation function and power spectrum of the complex envelope can be obtained by the CIR generated with graphs methods.

\section{A. Von Mises PDF of AOA}

We encounter non-isotropic scattering in various mobile radio channels, which strongly affects the correlation function and power spectrum of the complex envelope at Rx. Here, we use the versatile Von Mises angular distribution for modeling the non-uniform AoAs at Rx [13]. Based on this distribution, the correlation functions and power spectrum of the complex envelope at $\mathrm{Rx}$ are derived.

Let the random variable $\Theta$ represents the AoA of a multipath component received at Rx. Then the PDF of Von Mises for the scatter component of $\Theta, p_{\Theta}(\theta)$ is given by

$$
p_{\Theta}(\theta)=\frac{\exp \left[\kappa \cos \left(\theta-\theta_{p}\right)\right]}{2 \pi I_{0}(\kappa)}, \theta \in[-\pi, \pi)
$$

where $I_{0}(\cdot)$ is the zero-order modified Bessel function, $\theta_{p} \in[-\pi, \pi)$ accounts for the mean direction of AoA of scatter components, and $\kappa \geq 0$ controls the width of AoA of scatter components. Using $p_{\Theta}(\theta)$ for carrying out the expectation with respect to $\Theta$ yields

$$
\phi_{r r}^{s c a t}(\tau)=\frac{I_{0}\left(\sqrt{\kappa^{2}-4 \pi^{2} f_{m}^{2} \tau^{2}+j 4 \pi \kappa \cos \left(\theta_{p}\right) f_{m} \tau}\right)}{I_{0}(\kappa)}
$$

where $f_{m}$ is the maximum Doppler frequency.

\section{B. Non-Isotropic Scattering Model}

Fig. 2 plots a non-isotropic scattering model. In this graph the signal propagates in the following way: (1) The transmitter emits a signal via its outgoing edges to scatters; (2) Scatters receive the signal on its ingoing edges and re-emit the signal on the outgoing edges with no reflection among the scatters; (3) The signal observed at $\mathrm{Rx}$ can be expressed as the sum of the signals arriving via the ingoing edges. In our model, $\mathrm{Rx}$ is positioned on the train travelling at the speed of $350 \mathrm{~km} / \mathrm{h}$, and AoA of a multi-path component yields to the Von Mises distribution so that the position matrices of the scatterers obeying this distribution are generated. The transfer function of the propagation can be obtained in the frequency domain using equation (5). Then the channel spatialtemporal-frequency coefficients are obtained, and the CIRs

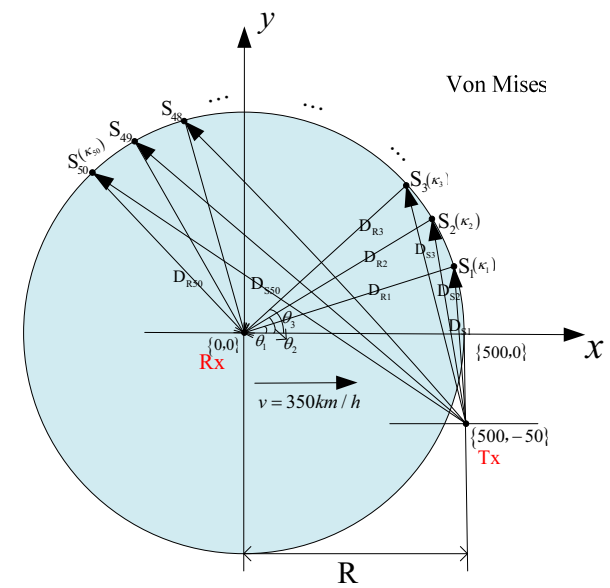

Fig. 2. A nonisotropic scattering model, AoAs of received signals are yield to the Von Mises pdf.

TABLE I

PARAMETER SETTING FOR THE SIMULATION

\begin{tabular}{cc}
\hline Parameters & Value \\
\hline Bandwidth & $100 \mathrm{MHz}$ \\
Carrier Frequency & $2.6 \mathrm{GHz}$ \\
Velocity of the train & $350 \mathrm{~km} / \mathrm{h}$ \\
Sampling Rate of channel & $2 \mathrm{KHz}$ \\
Model type & $\mathrm{SISO}$ \\
\hline
\end{tabular}

are calculated per snapshot. TABLE.I lists the parameters for the emulation. With the autocorrelation of CIRs $\left(\phi_{r r}^{\text {scat }}(\tau)\right)$, the maximum Doppler frequency is $f_{m}=842.60 \mathrm{~Hz}$, the unknown parameters $\left(\kappa, \theta_{p}\right)$ are estimated by the nonlinear least squares method: $\left(\hat{\kappa}, \hat{\theta}_{p}\right)=\arg \min _{\left(\kappa, \theta_{p}\right)} M S E_{\text {Simple }}$, where $M S E_{\text {Simple }}=n^{-1} \sum_{l=1}^{n}\left[\hat{\phi}_{r r}^{\text {scat }}\left(\tau_{l}\right)-\phi_{r r}^{\text {scat }}\left(\tau_{l}\right)\right]^{2}$.

TABLE II

THE COMPARISON OF INITIAL VALUES $\left(\kappa, \theta_{p}\right)$ With THE ESTIMATED $\operatorname{VALUES}\left(\hat{\kappa}, \hat{\theta}_{p}\right)$

\begin{tabular}{|c|c|c|c|c|}
\hline \multirow{2}{*}{ Record } & \multicolumn{2}{|c|}{ Initial value } & \multicolumn{2}{c|}{ Estimate value } \\
\cline { 2 - 5 } & $\kappa$ & $\theta_{p}$ & $\hat{\kappa}$ & $\hat{\theta}_{p}$ \\
\hline 1 & 20 & 45 & 20.04 & 37.92 \\
\hline 2 & 30 & 45 & 28.99 & 43.49 \\
\hline 3 & 40 & 45 & 33.08 & 51.36 \\
\hline 4 & 20 & 90 & 22.98 & 95.96 \\
\hline 5 & 30 & 90 & 27.70 & 92.64 \\
\hline 6 & 40 & 90 & 38.40 & 88.07 \\
\hline
\end{tabular}

The estimate results are shown in TABLE.II. Fig. 3 depicts the Doppler spectrum of the theoretical received signal and the results simulated by using graph models in the environment considered when $\left(\kappa, \theta_{p}\right)=\left(20, \frac{\pi}{4}\right)$, which illustrate explicitly that the estimated values $\left(\hat{\kappa}, \hat{\theta}_{p}\right)$ match well with the simulated values.

\section{Channel Modeling and Simulation for High-SPEEd RaILWAY SCENARIOS}

As described in Section II and Section III, the method proposed is applicable for generating synthetic propagation channels. Since most of the high-speed railways are constructed in the suburb, rural open area or viaduct above, scattering 


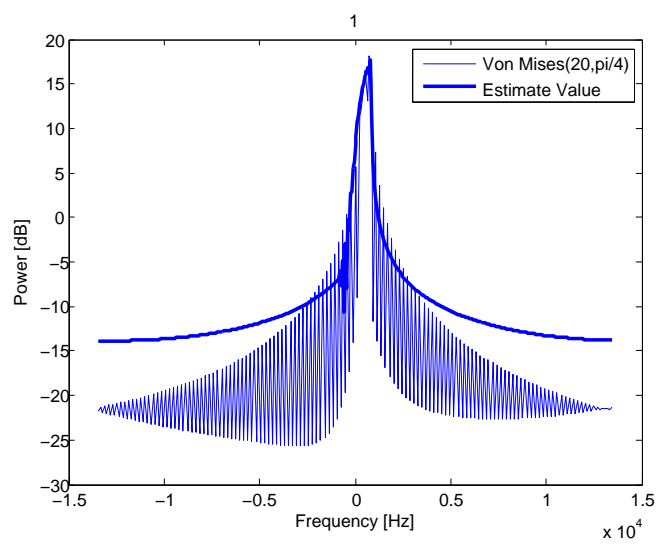

Fig. 3. The Doppler spectrum of the theoretical received signal and the results simulated by using graph models in the environment considered $\left(\kappa, \theta_{p}\right)=$ $\left(20, \frac{\pi}{4}\right)$.

characteristics of the high-speed railway at the same environment is relatively fixed compared with terrestrial cellular radio propagation channel. For the advantages of the propagation graph approach, the simulated CIRs can be generated directly based on the locations of the objects in a typical high-speed railway scenario and their electromagnetic properties.

\section{A. A Comparison between Measurement Data and Simulation Result}

We choose the cutting terrain as the measurement site, which is a typical scenario in HSR environment. Based on the inspection and actual measurement in cutting scenario, the base stations are positioned along the track, the distance between base station and high-speed rail line is close, furthermore, the height difference is noticeable. Therefore, radio wave propagation components under the cutting scenario can be divided into four categories: LOS component; dynamic scattering component (the opposite running high-speed rail trains); diffuse scattering components, eg., high-speed rail line pylons, both sides of the slope, vegetation.

The over-all time varying Power Delay Profiles (PDPs) during the entire measurement run in which the train travels through the coverage area are shown as figure 4 . From the enlarged detail view of Fig.4, we can see that there is a strong path component, whose delay is obviously increasing from zero relative to the dominant one with the period of approximately 0.89 s. Periodical propagation waves result from the scattering of the pylons along the railway at an interval distance of $50 \mathrm{~m}$. According to the investigation, the scattering component is caused by diffuse reflection region formed by the pylons evenly distributed on both sides of the tracks.

As described in Section II, the cutting scenario can be modeled by the directed-graph, where vertices represent telegraph pylons. The serial circular scattering regions resulted by the pylons are distributed uniformly on the track-side. $\mathrm{G}$ and $\mathrm{H}$ are the vertical distances from transmitter and pylon to the track, respectively. $\mathrm{K}$ denotes the coverage distance that the train passed through each scattering area. $\mathrm{P}$ and $\mathrm{Q}$ are the initial horizontal distances from train and pylon to the midpoint, respectively. And here $\mathrm{G}=92 \mathrm{~m}, \mathrm{H}=3.9 \mathrm{~m}, \mathrm{~K}=50 \mathrm{~m}, \mathrm{P}=600 \mathrm{~m}$, $\mathrm{Q}=575 \mathrm{~m}$. The other main parameters are listed in Table III.

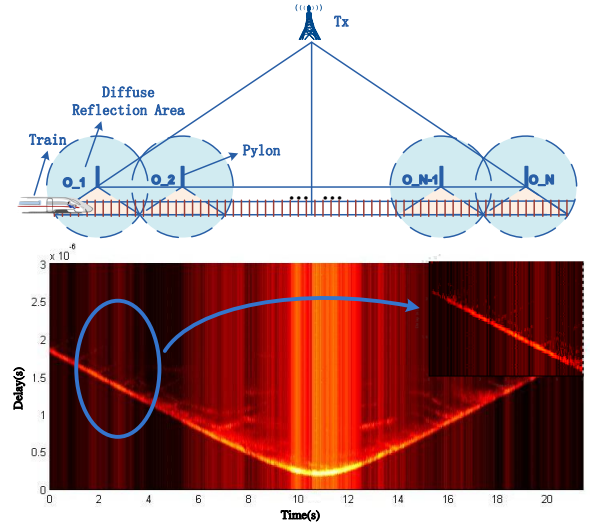

Fig. 4. Propagation environment abstract diagram in cutting scenario.

Fig.5 depicts the PDPs.

TABLE III

SYSTEM CONFIGURATION

\begin{tabular}{cc}
\hline Parameters & Value \\
\hline Center Frequency & $2.35 \mathrm{GHz}$ \\
Bandwidth & $50 \mathrm{MHz}$ \\
Mobile Station Velocity & $55.6 \mathrm{~m} / \mathrm{s}$ \\
Channel Sample Rate & $1968 \mathrm{~Hz}$ \\
\hline
\end{tabular}

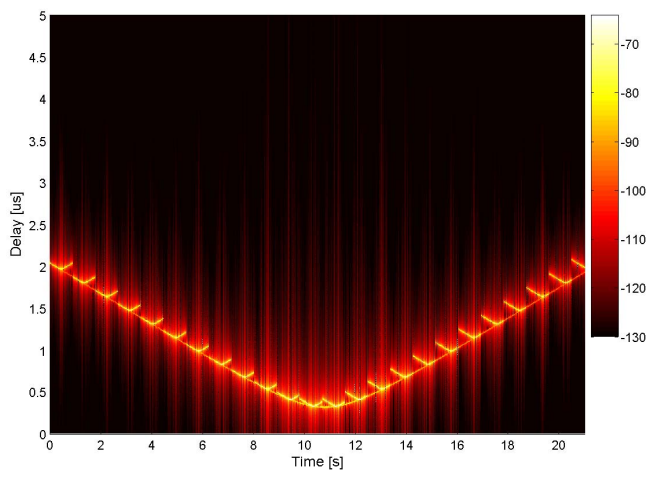

Fig. 5. CIR power variation in cutting scenario.

Fig.4 and Fig.5 qualitatively compare the results from measurement data and the propagation graph theory model. We can see that the study of channel modeling based on graph theory methods has a certain sense of the development of wireless communication.

\section{B. An Typical High-Speed Railway Scenario in Plains Envi- ronment}

Based on site spot investigation and data analysis of the existing measurements of high-speed railway, the radio waves component of high-speed railway scenario in plains environment can be mainly divided into the following two categories, LOS and NLOS component. NLOS component chiefly refers to discrete static scattering component including the reflection from the pylons along with the high-speed rail line and surrounding buildings which can be called Static Scatter (SS).

Fig. 6 depicts the graph model of the plains scene, where Tx is fixed at the location $500 \mathrm{~m}$ away from the first snapshot and Rx moves along the high-speed railway following the direction 


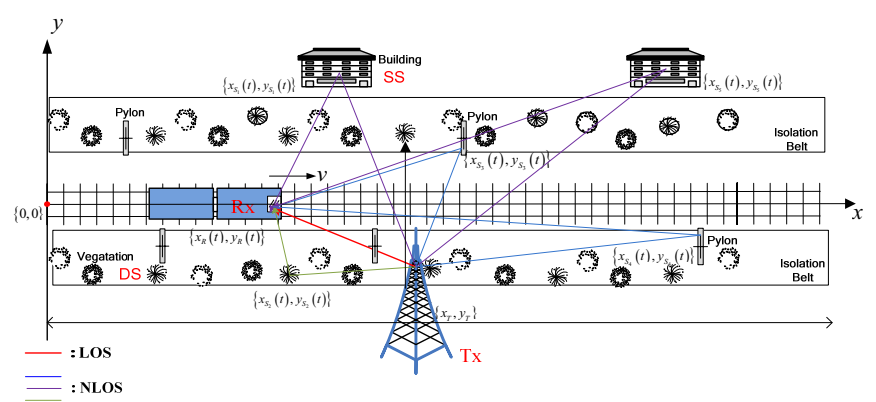

Fig. 6. The graph model of plain scenario.

shown by the arrows in the figure with a speed of $350 \mathrm{~km} / \mathrm{h}$, and the SS contains two scattering areas and five pylons. Based on the locations of Tx, Rx and scatters, 10000 snapshots of the graph are generated. The CIRs are then calculated by using the afore-mentioned method. The simulation of parameters are listed in TABLE.I.

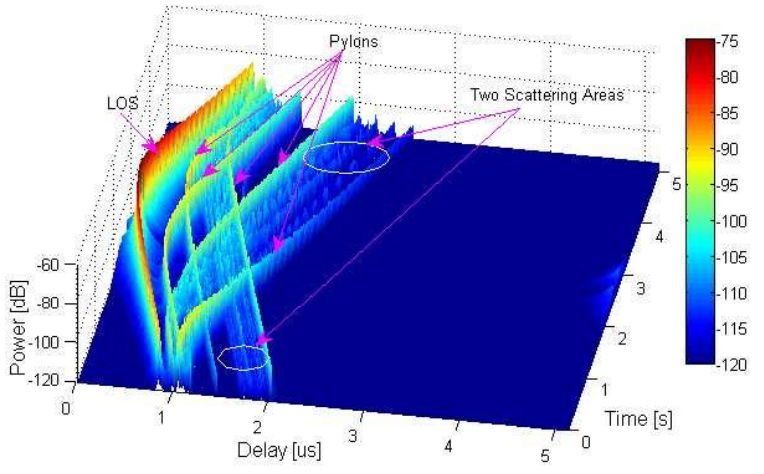

Fig. 7. PDP of the channel created in plain scenario.

Fig.7 depicts the PDP of the plain scenario. In Fig.7, we can see the power of LOS is dominant and it exists a convex trajectory. This phenomenon results from the fact that the train approaches and then leaves from Rx. The propagation distance changes with the transceiver separation. On the other hand, five strong echo waves from the pylons appear explicitly. Different reflection points derive from their different position. Finally, two multi-path bands are obviously due to the surrounding SS.

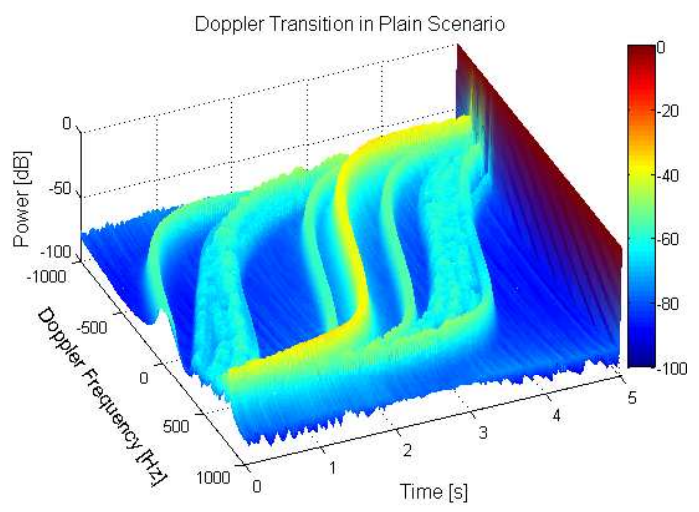

Fig. 8. Doppler transition in plain scenario with 10000 snapshots.

Muitple instantaneous Doppler characteristics are shown in Fig. 8. It is obvious that there is an apparent Doppler shift in the entire process, and the dominant Doppler lines sweep from the maximum to the minimum value not only when the train passes through the BS but in areas when the train crosses the scattering area and five pylons. We can conclude that rapid Doppler transition will occur if there is a scatterer near the rail.

\section{CONClusion}

In this paper, a novel geometry-based channel characterization for the high-speed railway is performed by using the graph modeling method. Von Mises is used to characterize the feasibility of this graph channel modeling method, and the results show that the setting of the parameters is reasonable. Furthermore, we compare the results of proposed modeling method with actual data, qualitative analysis enables the proposed method to have merit. According to extracted reasonable results on the time and frequency dispersion, it shows that scatterers along the rail-track not only bring multipath components in time-delay domain, but also produce the rapid Doppler transition when the train passes by.

\section{ACKNOWLEDGMENT}

The research was supported in part by the NSFC project under grant No.61371070 and No.61032002, open research fund of National Mobile Communications Research Laboratory Southeast University No.2012D07, and Fundamental Research Funds for the Central Universities under grant No.2013YJS016.

\section{REFERENCES}

[1] M. Failli, "Digital land mobile radio communications COST 207," Final Report, Commission of the European Communities, Tech. Rep., 1989.

[2] "Spatial channel model for Multiple Input Multiple Output (MIMO) simulations (Release 7)," 3GPP TR25.996 V7.0.0, 2007.

[3] K. Pekka, "WINNER II Channel Models part II Radio Channel Measurement and Analysis Results," 2007.

[4] L. Liu, C. Tao, J. Qiu, H. Chen, L. Yu, W. Dong and Y. Yuan, "Position-Based Modeling for Wireless Channel on High-Speed Railway under a Viaduct at $2.35 \mathrm{GHz}$," IEEE Journal on Selected Areas in Communications, vol. 30, no. 4, pp. 834-845, Apr. 2012.

[5] L. Liu, C. Tao, T. Zhou, J. Qiu, R. Sun and H. Chen, "A highly efficient channel sounding method based on cellular communications for highspeed railway scenarios," EURASIP Journal on Wireless Communications and Networking, Oct. 2012.

[6] T. Pedersen and B. H. Fleury, "A realistic radio channel model based on stochastic propagation graphs," in Proceedings 5th MATHMOD Vienna5th Vienna Symposium on Mathematical Modeling, vol. 1,2, Feb. 2006, p. 324, ISBN 3-901608-30-3.

[7] X. Yin, "Preliminary Study on Angular Small-Scale Cross-Correlation of Channels in NLOS Scenarios using Propagation Graphs," in The 5th International Workshop on Broadband MIMO Channel Measurement and Modeling, Beijing, China, Jun. 2011.

[8] T. Pedersen, "Modeling of Reverberant Radio Channels Using Propagation Graphs," May 2011.

[9] L. Tian, "Channel Simulation and Modeling Based on the Propagationgraph Theory for High-speed Railway Scenarios," in Proceedings of the Vehicular Technology Conference (VTC Spring), May 2012.

[10] T. Pedersen and B. Fleury, "Radio channel modelling using stochastic propagation graphs," in IEEE International Conference on Communications ICC '07, Jun. 2007, pp. 2733-2738.

[11] M. Patzold, Mobile Radio Channel (Second Edition), Wiley, 2012.

[12] T. Zhou, C. Tao, "High-Speed Railway Channel Measurements and Characterizations: A Review," in 1st Workshop on High Mobility Wireless Communications, Chengdu, China, Nov. 2012.

[13] A. Abdi, "A Parametric Model for the Distribution of the Angle of Arrival and the Associated Correlation Function and Power Spectrum at the Mobile Station," IEEE Transactions on Vehicular Technology, vol. 51, no. 3, May 2002 